\title{
DFT Study Of The Cyclization of 1,2-divinylbenzene And Derivatives
}

Enrique M. Cabaleiro-Lago ${ }^{(a)}$, Ángeles Peña-Gallego ${ }^{(b)}$, Jesús Rodríguez-Otero ${ }^{(b)}$.

(a) Departamento de Química Física, Facultade de Ciencias, Universidade de Santiago de Compostela, Campus de Lugo. Avda. Alfonso X El Sabio s/n 27002 Lugo, Galicia (Spain).

(b) Departamento de Química Física, Facultade de Química, Universidade de Santiago de Compostela, Avda. das Ciencias, s/n 15706 Santiago de Compostela, Galicia (Spain).

e-mail: qftkike@usc.es

\begin{abstract}
Electrocyclization reactions of 1,2-divinylbenzene and several related molecules were studied by performing density functional theory (DFT) calculations together with the $6-31+\mathrm{G}^{*}$ basis set. Reactants, products, and transition states for each reaction were localized and the IRC connecting reactants and products was also obtained. Magnetic properties were evaluated along the reaction path to elucidate the characteristics of the reactions studied with respect to their aromaticity and pericyclic character.

Though reactions $\mathbf{B}$ and $\mathbf{C}$ seem to be borderline cases between pericyclic and pseudopericyclic behaviour, the analysis of different magnetic properties allow us to conclude that all reactions studied are pericyclic.
\end{abstract}




\section{Introduction}

According to the original definition of Lemal, a pseudopericyclic reaction is a concerted transformation whose primary changes in bonding encompass a cyclic array of atoms, at one or more or which nonbonding and bonding atomic orbitals interchange roles.[1] Although Lemal's definition is seemingly clear, there is some ambiguity in it as the orbital description is not unique. As a consequence, no universally accepted clearcut, absolute criterion exists for distinguishing a pseudopericyclic reaction from a normal pericyclic one.

In this respect, magnetic criteria seemed to be the most useful ones. In a normal pericyclic reaction, a cyclic loop of orbitals is formed in the transition state, leading to aromaticity, whereas in pseudopericyclic reactions, the orbital disconnection prevents the transition state for being aromatic.[2,3] As a consequence, in a pericyclic reaction an aromaticity enhancement is appreciated in the vicinity of the transition state, whereas no enhancement of the aromaticity is observed in pseudopericyclic reactions.

Therefore, an analysis of magnetic properties (which reflect to some extent the presence of aromaticity) can be used as a criterion for distinguishing both reaction mechanisms.[3-5]. In the present work, we present an analysis of the evolution of magnetic properties along the reaction path for the reactions shown in figure 1, which correspond to the electrocyclizations of 1,2-divinylbenzene and several analogues.

Among these reactions, reaction $\mathbf{B}$ has been previously studied by Luo et al.,[6] who concluded that the reaction is compatible with a pseudopericyclic mechanism based in geometric (almost planar transition state) considerations. However, we have shown in previous works that the transition state geometry is not especially appropriate for distinguishing between the pericyclic/pseudopericyclic characters of a reaction. In fact, a similar reaction without the phenyl ring has been classified as pericyclic in a recent work.[7] We expect magnetic properties to reveal more clearly the character of this reaction. Also, reaction $\mathbf{C}$ was included because of its similarities with $\mathbf{B}$, whereas $\mathbf{D}$ is their all-carbon analogue.

[1] J. A. Ross, R. P. Seiders, D. M. Lemal, J. Am. Chem. Soc., 98 (1976) 43254327.

[2] H. E. Zimmermann, Acc. Chem. Res., 4 (1971) 272.

[3] R. Herges, H. Jiao, P. v. R. Schleyer, Angew. Chem. Int. Ed., 33 (1994) 1376.

[4] E. M. Cabaleiro-Lago, J. Rodriguez-Otero, J. M. Hermida-Ramon, J. Phys. Chem. A, 107 (2003) 4962-4966.

[5] J. Rodriguez-Otero; E. M. Cabaleiro-Lago, Chem. Eur. J., 9 (2003) 1837-1843. 


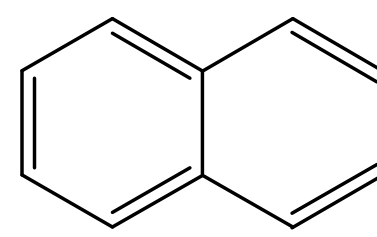

$\stackrel{\mathrm{A}}{\longrightarrow}$
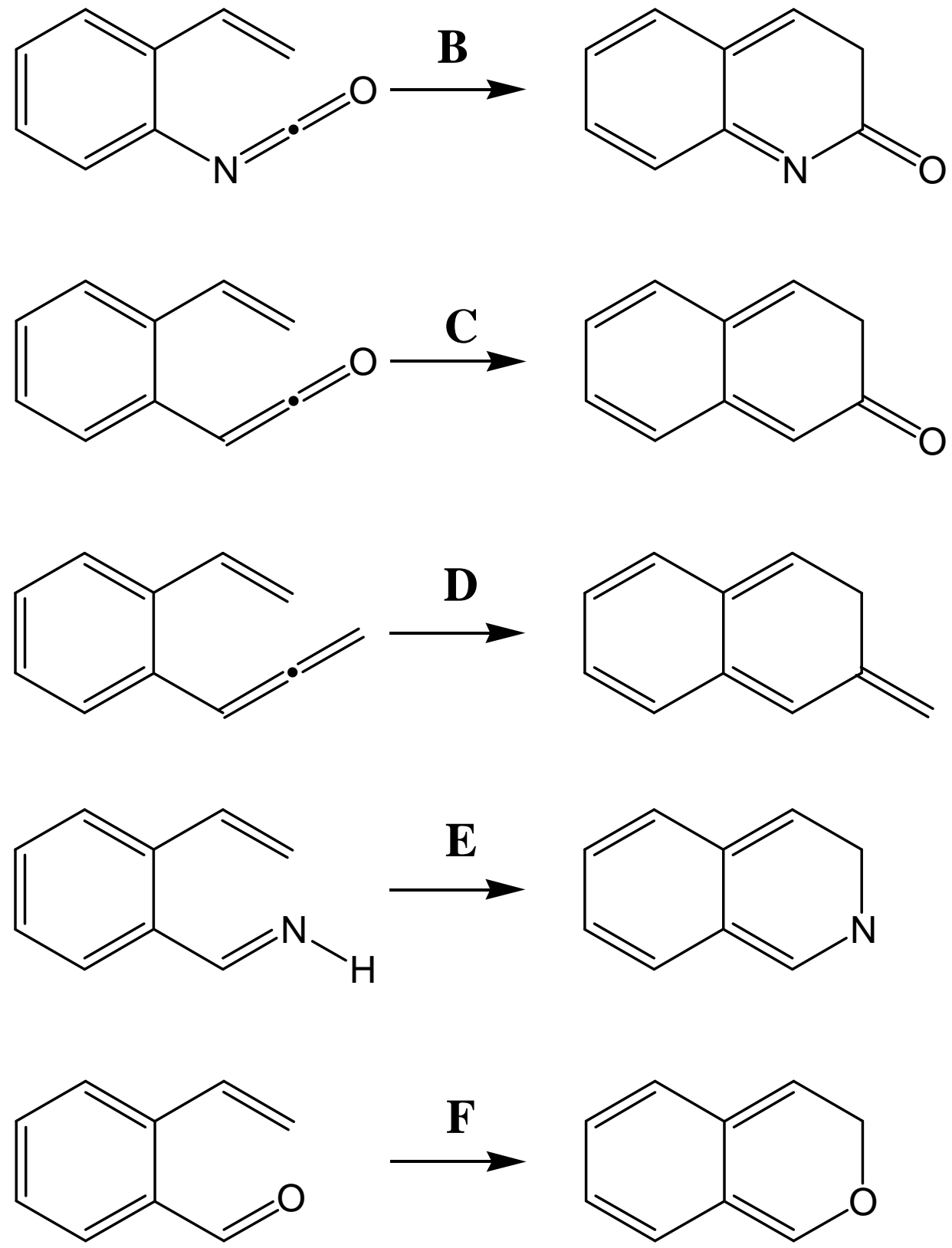

Figure 1. Reactions studied in this work. 


\section{Computational details}

The geometry of each stationary point was fully optimized using the $6-31+\mathrm{G}^{*}$ basis set and the B3LYP functional. All points were characterized as minima or transition structures by calculating the harmonic vibrational frequencies. Also, the pathway for each reaction was obtained by using the intrinsic reaction coordinate (IRC) with massweighted coordinates. The anisotropy of the magnetic susceptibility was then obtained at different points along the reaction path by computing the NMR shielding tensors at the B3LYP/6-31+G* level with the IGAIM method. Also, Nucleus Independent Chemical Shift (NICS) was obtained along the path evaluated at the same level of calculation.

\section{Results}

As noted from Table 1, all reactions present a considerable energy barrier. The largest barrier corresponds to reaction $\mathbf{A}$, amounting to $37.4 \mathrm{kcal} / \mathrm{mol}$. The introduction of the allene in reaction $\mathbf{D}$ diminishes the barrier to a value of $23.1 \mathrm{kcal} / \mathrm{mol}$. On the other side, it can be observed that reaction $\mathbf{B}$ exhibits a larger barrier than $\mathbf{D}$, reaching $26.5 \mathrm{kcal} / \mathrm{mol}$, whereas pseudopericyclic reactions usually show small barriers. Similar values are obtained for $\mathbf{E}$ and $\mathbf{F}$, and only for reaction $\mathbf{C}$ a smaller, still considerable, barrier of $13.9 \mathrm{kcal} / \mathrm{mol}$ is observed. On the other hand, all reactions are endothermic, except $\mathbf{C}$ and D. In summary, energetic considerations do not show appreciable differences among the reactions studied as to conclude about the pseudopericyclic character of any of them.

Figure 2 shows the variation of energy along the reaction path for the reactions studied. As it was commented above, it can be observed that all reactions behave similarly. 
Table 1. Energies (including ZPE) in $\mathrm{kcal} / \mathrm{mol}$ relative to the reactants for the reactions studied.

\begin{tabular}{|c|c|c|c|c|c|c|c|}
\hline$\Delta \mathbf{E}$ & $\mathbf{A}$ & 0.00 & 0.82 & & 4.66 & 37.39 & 3.39 \\
\hline$\Delta \mathbf{H}_{298}$ & & 0.00 & 1.81 & & 5.82 & 37.75 & 3.61 \\
\hline$\Delta \mathbf{E}$ & $\mathbf{B}$ & 0.00 & 0.79 & 1.25 & 2.52 & 26.50 & 13.04 \\
\hline$\Delta \mathbf{H}_{298}$ & & 0.00 & 0.75 & 1.19 & 2.50 & 25.53 & 12.20 \\
\hline$\Delta \mathbf{E}$ & C & 0.00 & 0.30 & 7.33 & 2.54 & 13.88 & -9.04 \\
\hline$\Delta \mathbf{H}_{298}$ & & 0.00 & 0.27 & 1.23 & 2.55 & 13.01 & -9.87 \\
\hline$\Delta \mathbf{E}$ & D & 0.00 & 1.21 & 1.40 & 4.80 & 23.14 & -11.55 \\
\hline$\Delta \mathbf{H}_{298}$ & & 0.00 & 1.66 & 1.88 & 5.43 & 22.80 & -11.95 \\
\hline$\Delta \mathbf{E}$ & $\mathbf{E}$ & 0.00 & 0.67 & 0.39 & 5.26 & 25.30 & 7.50 \\
\hline$\Delta \mathbf{H}_{298}$ & & 0.00 & 0.61 & 0.36 & 5.25 & 24.46 & 6.82 \\
\hline$\Delta \mathbf{E}$ & $\mathbf{F}$ & 0.58 & 1.22 & 0.00 & 3.61 & 29.57 & 20.77 \\
\hline$\Delta \mathbf{H}_{298}$ & & 0.61 & 1.20 & 0.00 & 3.57 & 28.69 & 19.98 \\
\hline
\end{tabular}




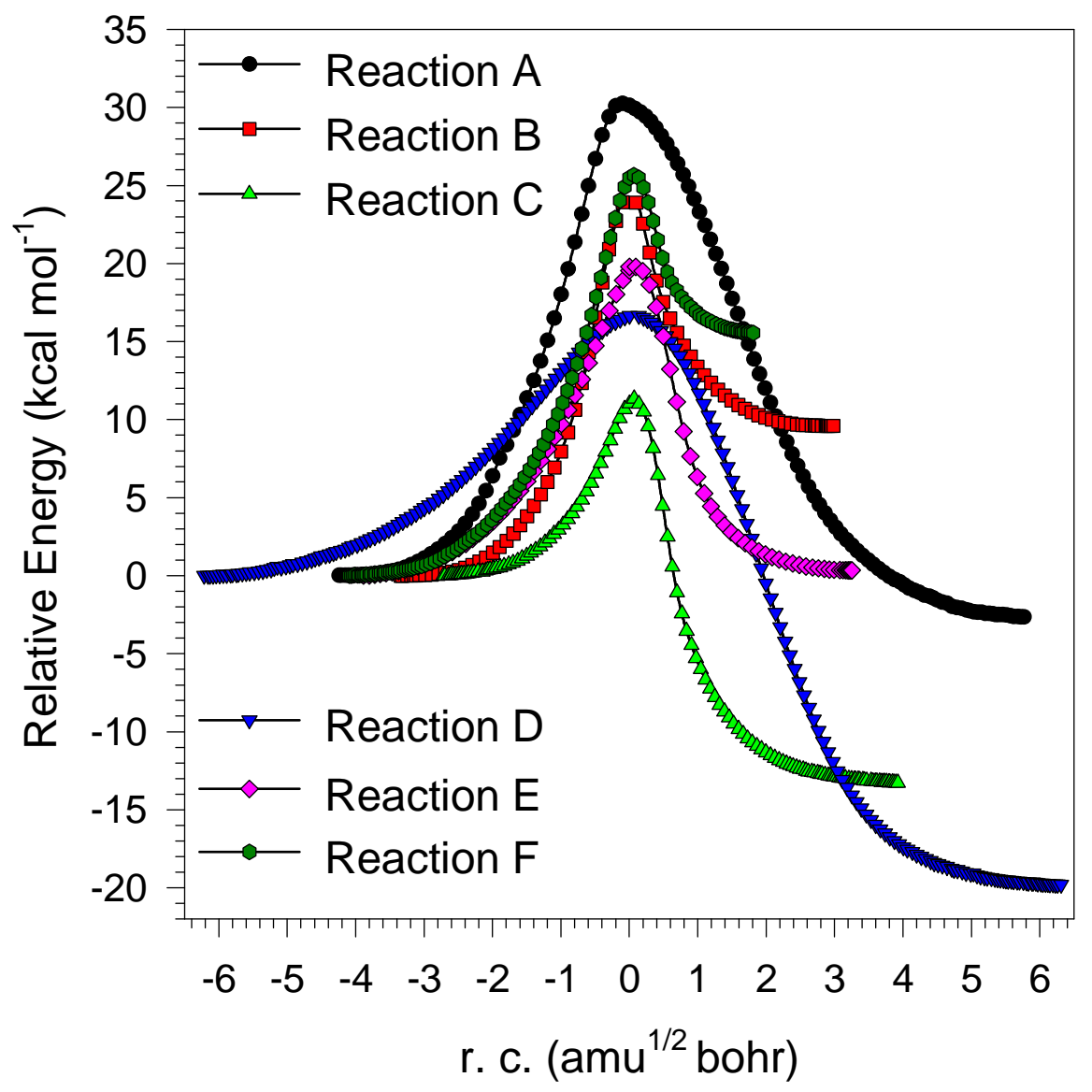

Figure 2. Energy variations along the reaction path for the reactions studied. 


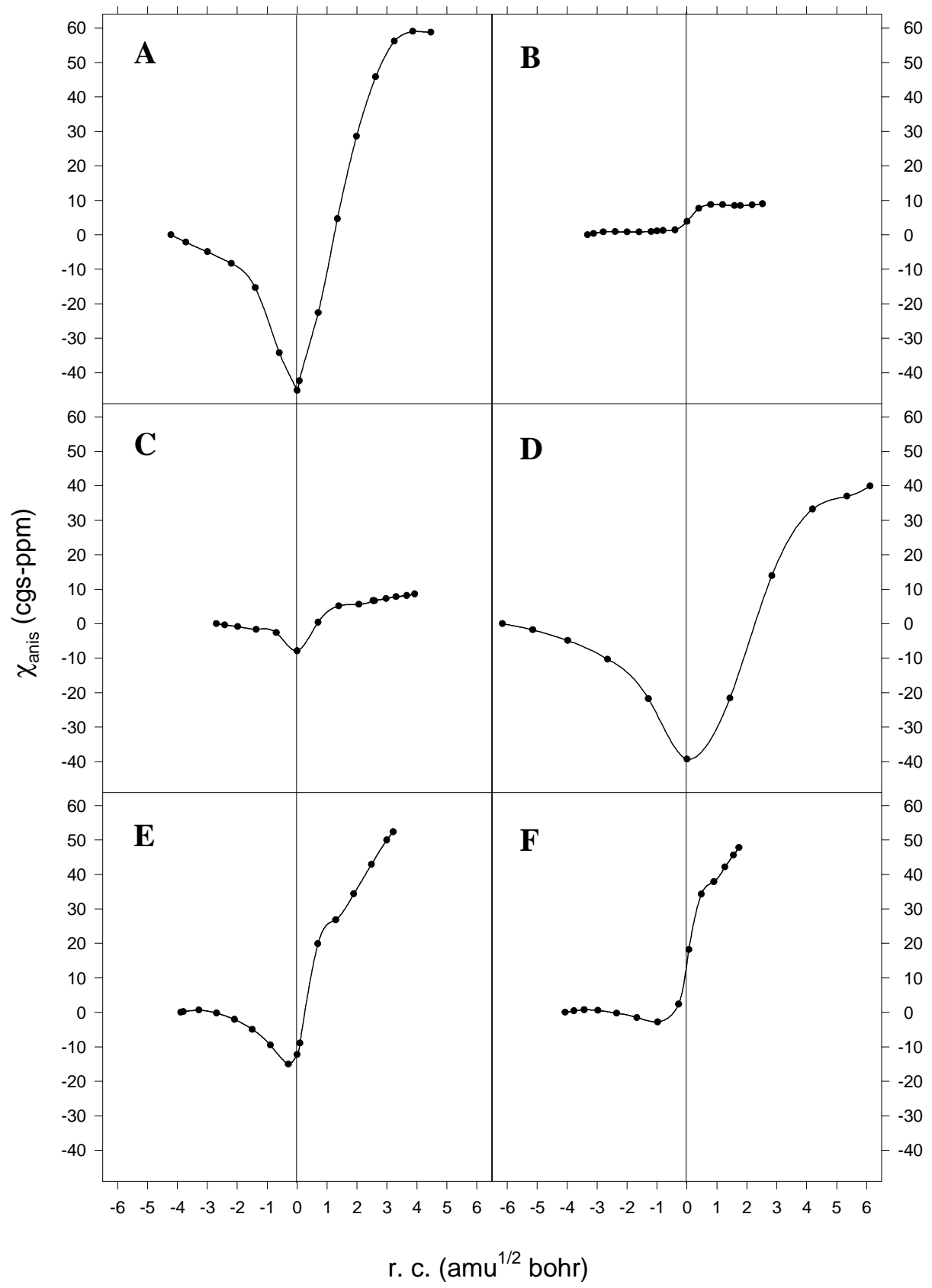

Figure 3. Variation of the anisotropy of the magnetic susceptibility along the reaction path relative to the reactants. 


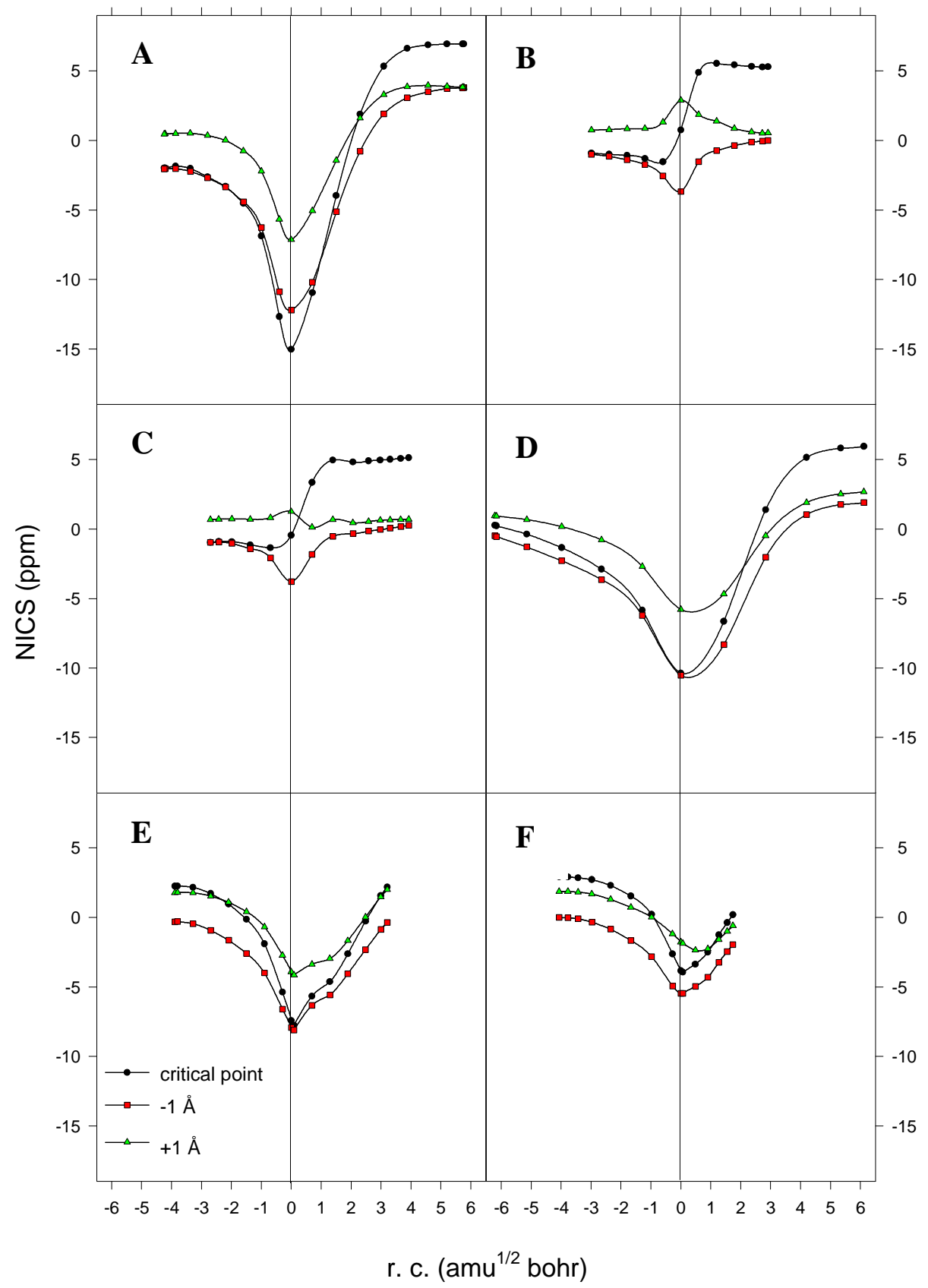

Figure 4. Variation of NICS along the reaction path as calculated in the points shown in figure 5 . 


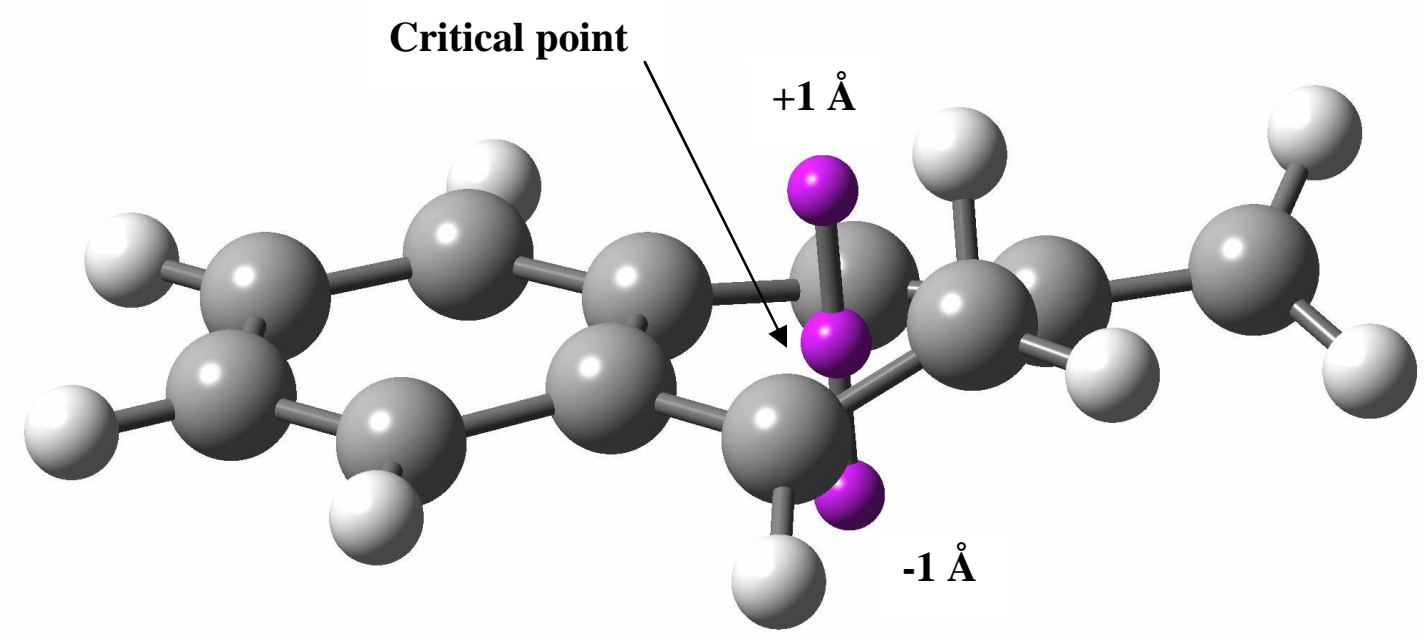

Figure 5. Points were NICS was calculated along the reaction path of each reaction studied.

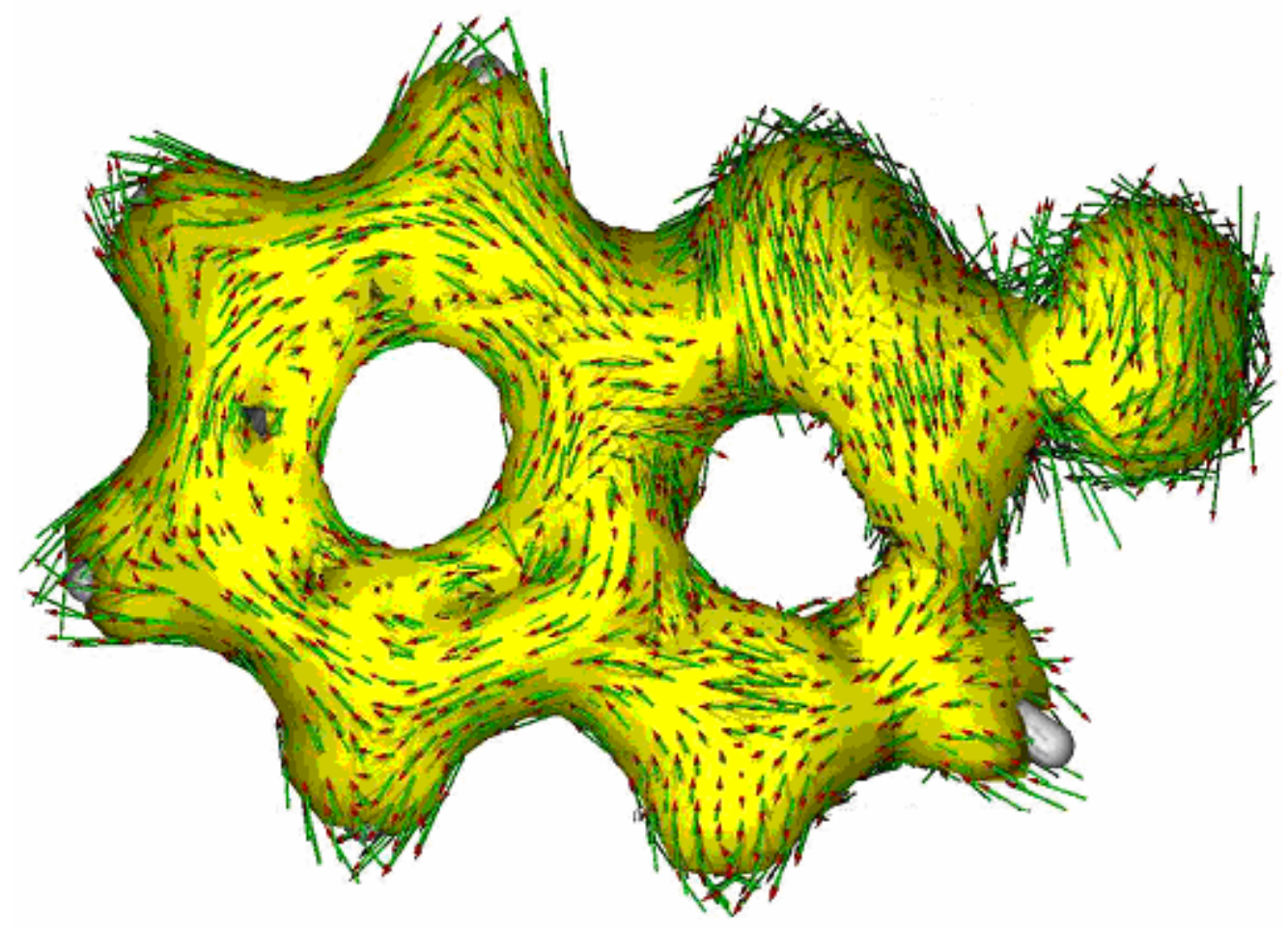

Figure 6. ACID plot for reaction $\mathbf{B}$ in the transition state. The magnetic field points to the reader. Isosurface value is 0.04 . 
Figure 3 shows the variation of the anisotropy of the magnetic susceptibility along the reaction path for the reactions studied. Considering that a pericyclic reactions evolves in such a way that aromaticity increases in the vicinity of the transition state, one should observe a minimum of the anisotropy of the magnetic susceptibility near the transition state. It should be a minimum because the enhancement must be present in both the cyclization and the ring opening (the two are pericyclic). Therefore, figure 3 indicates that reactions $\mathbf{A}, \mathbf{D}$ and $\mathbf{E}$ are clearly pericyclic, exhibiting well defined minima. On the other hand, reactions $\mathbf{C}$ and $\mathbf{F}$ show only small minima whereas reaction $\mathbf{B}$ presents no minimum at all. There appear unexpected differences between reactions $\mathbf{B}$ and $\mathbf{C}$ (one can expect both being similar) and also $\mathbf{E}$ and $\mathbf{F}$. However, it should be taken into account that the magnetic susceptibility is a global property and, as the cyclization goes on, the development of aromaticity on the new ring formed can be shielded by the aromaticity loss of the phenyl ring.

Therefore, a property like NICS (Nucleus Independent Chemical Shift), which is obtained at a given point (see figure 5) would be more appropriate. As shown in figure 4, all reactions exhibit minima near the transition state, indicating a development of an extra aromatic character. Only reactions $\mathbf{B}$ and $\mathbf{C}$ do not present a clear behaviour. In both cases, a small minimum is observed for the points placed at $-1 \AA$ under the ring critical point. However, this is the crucial point, because the reaction takes place on this side of the molecule, so there are the points placed at $-1 \AA$ those who represent how aromaticity evolves in the cyclization. As a consequence, we can say that reactions $\mathbf{B}$ and $\mathbf{C}$ maintain weak pericyclic characteristics, but they are not pseudopericyclic as for a pseudopericyclic reaction no minimum should be observed. In fact, we have studied recently reactions similar to $\mathbf{C}$ without the phenyl moiety, which show similar behaviour and were classified as pericyclic.[7]

Another criterion to assess aromaticity is the so called ACID (Anisotropy of the Induced-Current Density). In an acid plot one can observe the diatropic ring currents associated to an aromatic ring. Figure 6 shows the ACID plot for reaction $\mathbf{B}$ in the transition state where a diatropic ring current can be observed, indicating certain degree of aromaticity. The degree of delocalization can also be measured by means of the critical isosurface value (CIV); that is, the isosurface value at which the cyclic topology in ACID is lost to obtain an open one. The CIVs for the reactions studied are: 0.071; $0.054 ; 0.050 ; 0.068 ; 0.050 ; 0.044$ for $\mathbf{A}$ to $\mathbf{F}$. Therefore, all reactions show quite large values for CIV (for a typical pseudopericyclic reaction the value is usually <0.015) indicating pericyclic character. 